COO 264-548

COO 1701-11

\title{
REMARKS ON THE BETA DECAY OF HYPERONS
}

\author{
Reinhard Oehme, Roland Winston \\ and Augusto Garcia
}

The Enrico Fermi Institute

and The Department of Physics

The University of Chicago, Chicago, Illinois 60637

\author{
August, 1970 \\ Contracts No. AT $(11-1)-264$ \\ and AT $(11-1)-1701$
}

\section{This Lepal NOTICE}

This report was prepared as an account of .work sponsored by the United States Government. Neither the United States nor the United States Atomic Energy Commission, nor any of their employees, nor any of their contractors, subcontractors, or their employees, makes any warranty, express or implied, or assumes any legl liability or responsibility for the accuracy, comlegal liability or responsibility for the accuracy, completeness or usefulness of any information, apparatus, product or process disclosed, or represents that its use would not infringe privately owned rights. 


\section{DISCLAIMER}

This report was prepared as an account of work sponsored by an agency of the United States Government. Neither the United States Government nor any agency Thereof, nor any of their employees, makes any warranty, express or implied, or assumes any legal liability or responsibility for the accuracy, completeness, or usefulness of any information, apparatus, product, or process disclosed, or represents that its use would not infringe privately owned rights. Reference herein to any specific commercial product, process, or service by trade name, trademark, manufacturer, or otherwise does not necessarily constitute or imply its endorsement, recommendation, or favoring by the United States Government or any agency thereof. The views and opinions of authors expressed herein do not necessarily state or reflect those of the United States Government or any agency thereof. 


\section{DISCLAIMER}

Portions of this document may be illegible in electronic image products. Images are produced from the best available original document. 


\title{
REMARKS ON THE BETA DECAY OF HYPERONS*
}

Reinhard Oehme, Roland Winston

and Augusto Garcia

$$
\text { August, } 1970
$$

The Enrico Fermi Institute

and The Department of Physics

The University of Chicago, Chicago, Illinois 60637

\begin{abstract}
The formulae for spin and angular correlations in hyperon $\beta$-decay are brought into forms which can give specific information about the character of possible deviations from the universal SU(3) scheme. The usefulness of the proposed combinations is discussed qualitatively.
\end{abstract}

* Work supported in part by the U.S. Atomic Energy Commission. 
A concise description of semileptonic weak interactions has been given on the basis of the following assumptions. 1,2) 1. the weak vector current and the electromagnetic current consist of the appropriate components of a common octet current of $\mathrm{SU}(3), 2$. the axial-vector current is the same component of another octet current, and 3. there is a universal suppression factor for strangeness-changing transitions. In particular, the measured rates and the lepton-neutrino angular correlations of $\Delta S=l$ transitions appear to fit rather well into such a framework. However, more recently, very preliminary data on spin correlations for $\Lambda \rightarrow p+e^{-}+\bar{\nu}$ decays have become available, 3,4$)$ which can give a new and sensitive test of the assumptions 1-3. It is the purpose of this note to explore the possible implications of these measurements in a qualitative way. Specifically, we suggest a sum rule for the correlation coefficients which can give specific information.

We write the semileptonic interactions in the form

$$
\sqrt{2} \mathrm{~J}_{\alpha} \ell_{\alpha}^{+}+\mathrm{h} . \mathrm{c} \text {. }
$$

where $\mathrm{J}=\mathrm{V}+\mathrm{A}$, and $\ell$ is the familiar lepton current. The form factors appearing in the matrix element

$$
M=\left\langle p\left|v_{\alpha}+A_{\alpha}\right| \Lambda\right\rangle i \bar{e} \gamma_{\alpha}\left(1+\gamma_{5}\right) \nu_{e}
$$


are defined by*

$$
\begin{aligned}
& \left\langle p\left|v_{\alpha}\right| \Lambda>=\bar{u}_{p}\left(\gamma_{\alpha} f_{1}+\sigma_{\alpha \beta} q_{\beta} f_{2} / m_{\Omega}\right) u_{\Lambda},\right. \\
& \left\langle\mathrm{p}\left|\mathrm{A}_{\alpha}\right| \Lambda\right\rangle=\overline{\mathrm{u}}_{\mathrm{p}}\left(\gamma_{\alpha} \gamma_{5} \mathrm{~g}_{1}+\sigma_{\alpha \beta} \mathrm{q}_{\beta} \gamma_{5} g_{2} / \mathrm{m}_{\beta}\right) \mathrm{u}_{\alpha_{1}},
\end{aligned}
$$

where $q=p_{s}-p=p_{e}+p_{\nu}$. Terms involving $q_{\alpha}$ and $q_{\alpha} \gamma_{5}$ have been omitted because their contribution to $M$ is proportional to the electron mass. If time reversal invariance is assumed, the form factors $f\left(q^{2}\right)$ and $g\left(q^{2}\right)$ become real analytic functions, with $g_{2}$ being second class.

The transition rate is given by

$d u=\frac{G^{2}}{(2 \pi)^{5}} \frac{p_{e}^{2}\left(p_{e m a x}-p_{e}\right)^{2}}{\left[1+\frac{p_{e}}{m_{\curvearrowright}}(e \cdot \nu-1)\right]^{3}} \frac{m_{p}}{m_{\infty}}|M|^{2} d \Omega e^{d \Omega} \nu d p_{e^{\prime}}$

and we can write $|M|^{2}$ in the general form

$$
\begin{aligned}
& \xi\left\{l+a(\hat{e} \cdot \hat{v})+A \underset{m_{0}}{\sigma} \cdot \hat{e}+B \underset{m_{\infty}}{\sigma} \cdot \hat{v}+D \underset{m_{\sigma}}{\sigma} \cdot \hat{e x} \hat{\nu}\right. \\
& +A: \dot{m}_{n}^{\sigma} \hat{e}(\hat{e} \cdot \hat{\nu})+B^{\prime}{ }_{n}^{\sigma} \cdot \hat{v}(\hat{e} \cdot \hat{\nu}) \\
& \left.+D^{\prime} \sigma_{n} \cdot(\hat{\operatorname{ex}} \hat{\nu})(\hat{e} \cdot \hat{\nu})\right\},
\end{aligned}
$$

\footnotetext{
${ }^{\star}$ We use the notation $p=\left(p_{m}, i p_{0}\right)$, etc., with hermitian
} $\gamma$-matrices. 
where $\hat{e}$ and $\hat{v}$ are unit vectors in the directions $p_{m}$ and $\underline{p} v$, and $\sigma$ is the polarization vector of the hyperon. The coefficients are functions of $p_{e}$ and $(\cos \theta)^{2}, \cos \theta \equiv(\hat{e} \cdot \hat{\nu})$, and they can be expressed as bilinear forms of the functions $f_{i}$ and $g_{i}$. The primed terms in Eq. (5) are absent in the allowed approximation.

In view of limited experimental information which is now becoming available, we are mainly interested in correlations integrated over $\mathrm{p}_{e}$ and averaged over the appropriate angle. We evaluate these quantities as expansions in $\Delta m / m=\left(m_{\wedge}-m_{p}\right) / m$ $\approx 0.16$, and we reproduce in the following only terms of zeroth and first order; an exception is made for some special second order contributions to be mentioned later.

In terms of the coefficients appearing in Eq. (5), the actually measured quantities presently available are the following: the $\hat{e} . \hat{v}$ correlation

$$
a_{0}=\left(\bar{a}-\frac{3}{2} \frac{\Delta m}{m}\right)\left(1-\frac{\Delta m}{2 m} \bar{a}\right)^{-1},
$$

the $\underset{\text { ns. }}{\sigma} . \hat{e}$ correlation

$$
\alpha=\left(\overline{\mathrm{A}}+\frac{1}{3} \overline{\mathrm{B}}^{\prime}-\frac{\Delta \mathrm{m}}{2 \mathrm{~m}} \overline{\mathrm{B}}\right)\left(1-\frac{\Delta \mathrm{m}}{2 \mathrm{~m}} \overline{\mathrm{a}}\right)^{-1},
$$

the $\sigma_{n} \cdot \hat{v}$ correlation

$$
\beta=\left(\bar{B}+\frac{l}{3} \bar{A}^{\prime}-\frac{\Delta m}{2 m} \bar{A}\right)\left(1-\frac{\Delta m}{2 m} \bar{a}\right)^{-1},
$$

and the rate, which is proportional to

$$
<\xi>\left(1-\frac{\Delta m}{2 m} \bar{a}\right)
$$


In Eqs. (6-9), the bar indicates the average over the spectrum and also over the angle. We have, for example

$$
\overline{\mathrm{A}}=\frac{\langle\xi \AA\rangle}{\langle\xi\rangle}
$$

where we use the notation

$$
\langle F\rangle=\int_{0}^{p_{e m a x}} p_{e} p_{e}^{2}\left(p_{e m a x}-p_{e}\right)^{2} \frac{1}{2} \int d \cos \theta F\left(p_{e}, \cos ^{2} \theta\right) \cdot 30 p_{e m a x}^{-5}
$$

The measured correlations determine three independent combinations of the coefficients appearing in Eq. (5). For the purpose of our discussion, we find it convenient to introduce the expressions

$$
\begin{aligned}
& \left(\overline{\mathrm{B}}+\frac{1}{3} \overline{\mathrm{A}}^{\prime}\right)-\left(\overline{\mathrm{A}}+\frac{1}{3} \overline{\mathrm{B}}^{\prime}\right)=\left(1-\frac{\Delta \mathrm{m}}{2 \mathrm{~m}}(1+\overline{\mathrm{a}})\right)(\beta-\alpha), \\
& \left(\overline{\mathrm{B}}+\frac{1}{3} \overline{\mathrm{A}}^{\prime}\right)+\left(\overline{\mathrm{A}}+\frac{1}{3} \overline{\mathrm{B}}^{\prime}\right)=\left(1+\frac{\Delta \mathrm{m}}{2 \mathrm{~m}}(1-\overline{\mathrm{a}})\right)(\beta+\alpha),
\end{aligned}
$$

and

$$
\frac{1-\bar{a}}{1+\bar{a}}
$$


In writing Eqs. (10) and (11), we have made use of the fact that the coefficients $\bar{A}^{\prime}$ and $\bar{B}^{\prime}$ are of first order in $\Delta \mathrm{m} / \mathrm{m}$.

We now construct a very specific combination of correlation coefficients which is of particular interest for the interpretation of experiments. We consider the ratio

$$
\Sigma=\frac{\left(\overline{\mathrm{B}}+\frac{1}{3} \overline{\mathrm{A}}^{\prime}\right)-\left(\overline{\mathrm{A}}+\frac{1}{3} \overline{\mathrm{B}}^{\prime}\right)-(1-\overline{\mathrm{a}})}{1+\overline{\mathrm{a}}},
$$

where

$$
\begin{gathered}
\langle\xi\rangle(1+\bar{a}) \Sigma= \\
\frac{\Delta m}{m} \frac{2}{3}\left\{\left|f_{1}\right|^{2}+\left|g_{1}\right|^{2}+2 \operatorname{Re}\left(f_{1} f_{2}^{*}\right)+2 \operatorname{Re}\left(g_{1} g_{2}^{*}\right)\right\} \\
+\left[-\left(\frac{\Delta m}{m}\right)^{2} \frac{2}{2}\left(5\left|f_{2}\right|^{2}+11\left|g_{2}\right|^{2}\right)\right]+0\left(\left(\frac{\Delta m}{m}\right)^{2}\right) .
\end{gathered}
$$

and

$$
\begin{gathered}
<\xi>(1+\bar{a})=2\left(\left|\mathrm{f}_{1}\right|^{2}+\left|\mathrm{g}_{1}\right|^{2}\right)\left(1+\frac{\Delta \mathrm{m}}{\mathrm{m}}\right) \\
+\left[\left(\frac{\Delta \mathrm{m}}{\mathrm{m}}\right)^{2} \frac{2}{7}\left(\left|\mathrm{f}_{2}\right|^{2}-\left|\mathrm{g}_{2}\right|^{2}\right)\right]+0\left(\left(\frac{\Delta \mathrm{m}}{\mathrm{m}}\right)^{2}\right) .
\end{gathered}
$$

As mentioned before, we have included only those second order terms which depend solely upon $f_{2}$ and $g_{2}$. We ignore the $q^{2}$-dependence of form factors as a small, second order correction.* The combination $\Sigma$ is constructed so as to vanish in the allowed approximation. Hence $\Sigma$ is zero within errors for

* We also omit radiative corrections, which may be permissable for our qualitative considerations. 
neutron $\beta$-decay.* For the $\Lambda-\beta$-decay, we may use the assumptions 1. -3 . of Cabibbo, which yield ${ }^{7}$

$$
g_{1} \approx 0.74 f_{1}, f_{2}=\frac{1}{2} \mu_{p} f_{1}, g_{2}=0,
$$

and hence

$$
\Sigma \approx 0.10
$$

Besides being first forbidden, the combination $\Sigma$ has the important property that it does not contain any interference term between $\mathrm{f}_{1}$ and $\mathrm{g}_{1}$.

For our later discussions, we also need the other independent combinations of correlation coefficients: Let us take the expressions $(12-13)$ and

$$
\Pi=\frac{\left(\overline{\mathrm{B}}+\frac{1}{3} \overline{\mathrm{A}}^{\prime}\right)+\left(\overline{\mathrm{A}}+\frac{1}{3} \overline{\mathrm{B}}^{\prime}\right)}{1+\overline{\mathrm{a}}}
$$

where

$$
\begin{aligned}
& <\xi>(1-\bar{a})=4\left|g_{1}\right|^{2}-\frac{\Delta m}{m} 8 \operatorname{Re}\left(g_{1} g_{2}^{*}\right) \\
& +\left[\left(\frac{\Delta m}{m}\right)^{2} \frac{2}{7}\left(3\left|f_{2}\right|^{2}+13\left|g_{2}\right|^{2}\right)\right]+0\left(\left(\frac{\Delta m}{m}\right)^{2}\right),
\end{aligned}
$$

and

$$
\begin{aligned}
& <\xi>(1+\bar{a}) \quad \Pi=4 \operatorname{Re}\left(f_{1} g_{1}^{*}\right) \\
& +\frac{\Delta m}{m} \frac{4}{3} \operatorname{Re}\left(2 f_{1} g_{1}^{*}-g_{1} f_{2}^{*}-f_{1} g_{2}^{*}\right) \\
& +\left[\left(\frac{\Delta m}{m}\right)^{2} \frac{32}{21} \operatorname{Re}\left(f_{2} g_{2}^{*}\right)\right]+0\left(\left(\frac{\Delta m}{m}\right)^{2}\right) .
\end{aligned}
$$

We note that, in contrast to $\Sigma$, the combination $\Pi$ is dependent only upon interference terms, in particular the main term is proportional to $\mathrm{f}_{1} g_{1}$. With the Cabibbo values (16), we obtain $\Pi \approx 0.86$

*For the relevant data see Ref. 6 . 
In order to illustrate the possible applications of our combinations let us take a specific example. We suppose that the correlation experiments give a result for $\Sigma$ which is negative." Then we see from Eq. (14) that 1) a simple adjustment of the $g_{1} / f_{1}$ ratio, or the introduction of a time reversal invariance violating phase ${ }^{13}$ ) between $g_{1}$ and $f_{1}$, would generally not lead to a negative value for $\Sigma$. 2) what would apparently be required are rather large values of $\left|f_{2}\right|$ and/or $\left|g_{2}\right|$. In particular, these form factors should have negative sign relative to $f_{1}, g_{1}$ because the $\mathrm{f}_{2}{ }^{2}$ and $\mathrm{g}_{2}{ }^{2}$ terms in $\Sigma$ have very small coefficients.

*We give here some very preliminary data obtained from recent correlation experiments. It is important to note that these are meant only as an example for the purpose of illustrating the possible usefulness of our combinations $\Sigma, \pi$, etc..For the actual experimental results, the forthcoming publications of the experimental groups should be consulted.

$$
\begin{aligned}
a_{0}=-0.08 \pm 0.11 & & (\text { Ref.8,9,10,11) } \\
\alpha=0.18 \pm 0.13 & & (\text { Ref. 3,10,12) } \\
\alpha=0.10 \pm 0.10 & & (\text { Ref. 4) } \\
\beta=0.73 \pm 0.19 & & (\text { Ref. 3,10). }
\end{aligned}
$$

From these numbers we obtain:

$$
\begin{aligned}
\Sigma & =0.26 \pm 0.19 \\
\Pi & =0.79 \pm 0.19 \\
\frac{1-\bar{a}}{1+\bar{a}} & =0.72 \pm 0.14
\end{aligned}
$$


From the universal SU(3) scheme, we find approximately

$$
\begin{aligned}
\Sigma & =0.10 \\
I & =0.86 \\
\frac{1-\bar{a}}{1+\bar{a}} & =0.61
\end{aligned}
$$


From Eqs. (15) and (18), we see that the rate and the ev angular correlation are relatively insensitive to $f_{2}$, and since these quantities agree reasonably well with the Cabibbo scheme, it may be suggestive to leave $g_{2}$ small or zero and allow a negative $\mathrm{f}_{1} \mathrm{f}_{2}$ term in $\Sigma$ in order to obtain a negative value for this expression. * However, as seen from Eq. (19), such a choice of $\mathrm{f}_{2}$ would give a positive contribution to the combination $\Pi$ in Eq. (17). This may lead to a value for $\Pi$ which is too large in comparison with experiment.

As far as $\sum$ and $\Pi$ are concerned, a negative value of $g_{2}$ relative to $g_{1}$ and $f_{1}$ has effects rather similar to the corresponding choice of $f_{2}$ discussed above, but the $g_{1} g_{2}$ term appears in the expression $(1-\bar{a})(1+\bar{a})^{-1}$ with a relatively large coefficient, and hence a complete reevaluation of the fit for the rates and the ev-correlation would be required.

of interest is also the possibility of a large, time reversal invariance violating second class contribution . 14) For $\Lambda$ $\beta$-decay, this can be realized with a form factor $g_{2}$ which has a phase of $90^{\circ}$ relative to the first class terms. Except for the T-violating terms proportional to $\sigma \cdot \hat{\sigma} . \hat{\mathrm{e}} \mathrm{x} \hat{\mathrm{v}}$ in $\mathrm{Eq}$. (5), an imaginary $g_{2}$ contribution can have an effect only in the order $(\Delta \mathrm{m} / \mathrm{m})^{2}$. Although a very large $\left|g_{2}\right|^{2}$ term in $\Sigma$ could give a negative value, the corresponding contributions to II and to Eq.

${ }^{\star}$ For $\Sigma=-0.3$, about $\mathrm{f}_{2} \sim-4 \mathrm{f}_{\mathrm{l}}$ is required; for $\Sigma=0$, we find $\mathrm{E}_{2} \sim-0.8 \mathrm{f}_{1}$. 
(12) may create additional discrepancies unless sizable changes are made in the real form factors.

At present, in view of the uncertainty of the experimental results, we do not want to consider complete solutions of the system of equations (14), (15), (18) and (19). Such solutions should be looked for with relations complete up to second order in $\Delta \mathrm{m} / \mathrm{m}$, or with the exact formulae*.

The example discussed above is meant to give an indication for the possible usefulness of the combinations $\Sigma$ and $\pi$ in the analysis of hyperon $\beta$-decay experiments. In particular, these expressions are very helpful for the analysis of possible deviations from the SU(3) scheme described in the introduction. They show that these hyperon $\beta$-decay experiments provide a most interesting independent test of the SU(3) scheme and perhaps even of the current-current form of the semileptonic interaction, at least as far as strangeness-changing transitions are concerned.

If the final analysis of the data should indicate that there are larger deviations from the universal SU(3) scheme, then such a result may also have implications for the understanding of the common suppression factor for $\Delta S=I$ transitions. ${ }^{15)}$ Using the SU (3) properties of the weak current, and the SU(3) $x \mathrm{SU}(3)$. algebra, we have shown some time ago that this suppression cannot be understood as a strong interaction effect. ${ }^{16)}$ However,

* Another source of information is of course the shape of the electron spectrum. 
if there is other evidence for large symmetry breaking effects, these assumptions may have to be reassessed.

The $\Lambda-\beta-$ decay is distinguished from the neutron $\beta$-decay by a much larger $Q$-value and by the change of strangeness. It would be most interesting to have spin-correlation experiments for $\Delta S=O$ transitions with larger Q-values. 


\section{References}

1. N. Cabibbo, Phys. Rev. Letters 10, 531 (1963).

2. M. Gell-Mann and M. Levy, Nuovo Cimento 16, 706 (1960);

M. Gell-Mann, Physics 1,63 (1964).

3. Argonne, Chicago, Ohio State, Washington University Collaboration, (private communication).

4. CERN-Heidelberg Collaboration (private communication).

5. J.M. Watson and R. Winston, Phys. Rev. 181, 1907 (1969); D.R. Harrington, Phys. Rev. $120,1482(\overline{1960}) ;$ P.S. Desai, Phys. Rev. 179,1327 (1969); these papers contain many further references.

6. M.T. Burgy, V.E. Krohn, T.B. Novey, G.R. Ringo and V.L. Telegdi, Phys. Rev. 120, 1829 (1960).

7. H. Filthuth, Talk at the Topical Conference on Weak Interactions, CERN, Geneva, 1969.

8. J.E. Maloney and B. Sechi-zorn, Phys. Rev. Letters 23, 425 (1969).

9. R.J. Loveless, J. Canter, J.A. Cole, J. Lee-Franzini, P. Franzini, Bulletin Am Phys. Soc. 14, 519 (1969).

10. V.G. Lind, T.O. Binford,M.L. Good and D. Stern, Phys. Rev. 135 , B 1483 (1964).

11. C. Baglin, V. Brisson, A. Rousset, J. Six, H.H. Bingham et. al., Nuovo Cimento 35, 977 (1965).

12. J. Barlow, I.M. Blair, G. Conforto, M.I. Ferrero, C. Rubbia, J.C. Sens, P.J. Duke, A.K. Mann, Physics Letters 18, 64 (1965).

13. S.L. Glashow, Phys. Rev. Letters 14, 35 (1965); J.C. Pati, Phys. Rev. Letters 20, 812 (1968).

14. N. Cabibbo, Phys. Letters 12, 137 (1964).

15. R. Oehme, Phys. Rev. Letters 12, 550, 604 (1964); Ann. of Physics (N.Y.) 33, 108 (1965).

16. R. Oehme and G. Segre, Phys. Letter's 11, 94 (1964). 
One of us (RO) would like to thank Professor J. Heintze for a most interesting and helpful discussion concerning the CERN-Heidelberg experiment.

The last-named author is indebted to SEDICT, Instituto Politecnico Nacional, Mexico, for partial support. 